\title{
ELECTROCHEMICAL DETERMINATION OF CHLOROACETIC ACID
}

\author{
NAMAL PRIYANTHA * and SARATH MALAVIPATHIRANA \\ Department of Chemistry, University of Peradeniya, Peradeniya.
}

(Received: 10 August 1995; accepted: 01 March 1996)

\begin{abstract}
Glassy carbon electrodes coated with 5,10,15,20-tetraphenylporphyrinato iron(III) chloride can electrocatalytically reduce chloroacetic acid. The catalytic current obtained in steady-state amperometric experiments is linearly proportional to the bulk concentration of chloroacetic acid between lower and upper detection limits, which demonstrates the analytical utility of the coated electrode.
\end{abstract}

Key words: Electrocatalysis, chloroacetic acid, electrochemical detection, metalloporphyrins.

\section{INTRODUCTION}

Among commonly used agropesticides, organochlorine compounds have a long lifetime,${ }^{1}$ and there is a demand to develop accurate analytical methodology to detect them. Existing analytical techniques such as gas chromatography and high performance liquid chromatography have several drawbacks that may be overcome by electroanalytical techniques, that are precise, relatively inexpensive and selective. ${ }^{2-4}$ Electrochemical reactions of organic molecules show slower reaction rates than those of inorganic ions due to the relatively larger molecular size. However, many organic reactions can be facilitated by introducing suitable electrocatalysts. Some electrocatalysts consist of a metal centre where the oxidation state of the metal is changed during the electrochemical process. For instance, metalloporphyrins have been used to enhance the reaction rate of the reduction of oxygen ${ }^{5,6}$ and organohalides.$^{7,8}$ Metalloporphyrins have also been used to catalyze the oxidation of some clinically significant molecules ${ }^{9}$ and sulfur containing compounds. ${ }^{10}$

Because of poor solubility in aqueous media, chemical investigations of metalloporphyrins were limited to nonaqueous media . ${ }^{11}$ As a result, chemical sensors based on such catalytic materials were not developed until recently. However, this problem has been overcome after analytical applications of chemically modified electrodes were introduced. Modified electrodes have been prepared by incorporating a thin film of metalloporphyrin molecules onto the surface of an electrode, or immobilizing them in a supporting matrix which is deposited on an electrode surface. ${ }^{12,13}$. Such modified electrodes are completely

*. Corresponding author:

Abbreviations: ECE - Electrochemical-chemical-eletrochemical; GC - Glass Carbon; SCE - Saturated Calomel electrode; Fe(III) TPPCl - 5,10,15,20-tetraphenyl porphyrinatoiron(III) chloride. 
different from unmodified (bare) electrodes and they display the properties of the incorporated material. Glassy carbon electrodes modified with metalloporphyrins have already been used as analytical sensors for several environmental pollutants and clinical substances. ${ }^{9,14}$

We report here on the use of glassy carbon disk electrodes modified with a thin coating of 5,10,15,20-tetraphenylporphyrinatoiron(III) chloride as an amperometric sensor for chloroacetic acid. Catalytic efficiency of the metalloporphyrin on chloroacetic acid is demonstrated using cyclic voltammetric techniques, and the proposed sensor is characterized by steady-state amperometric experiments. Chloroacetic acid is environmentally significant because its sodium salt is a common post-emergence contact herbicide that can affect on a wide range of annual weeds at the seedling stage. It is also used in combination with 'atrazine' to control total weed on noncrop land. ${ }^{15}$

\section{METHODS AND MATERIALS}

Materials: The compound, 5,10,15,20-tetraphenylporphyrinatoiron(III) chloride $[\mathrm{Fe}(\mathrm{III}) \mathrm{TPPCl}]$ and chloroacetic acid were purchased from Aldrich, USA and $\mathrm{BDH}, \mathrm{UK}$, respectively. Dichloromethane (AR) was distilled prior to use. All electrolyte solutions were prepared using lithium chloride (AR) supporting electrolyte in either freshly distilled water or the acetonitrile/water mixed solvent system of an appropriate proportion. Acetonitrile was used as received in preparation of these solutions.

Instrumentation: All cyclic voltammetric and amperometric experiments were performed with an Oxford Instruments potentiostat and recorded on a Yew Instruments Model $3022 \mathrm{X}-\mathrm{Y}$ recorder. A three electrode system consisting of a glassy carbon disk working (Bioanalytical Systems, USA), platinum wire counter and a saturated calomel (SCE) reference was used in all electrochemical measurements. All potentials are reported with respect to SCE. Deaeration of the electrochemical cell was accomplished by sparging with nitrogen, and the volume of the solution in the electrochemical cell was always $25.0 \mathrm{~mL}$.

Coating Procedure: The metalloporphyrin coating solution of $5 \times 10^{-4} \mathrm{M}$ was prepared in distilled dichloromethane. For the initial coating, the glassy carbon disk electrode was cleaned by rubbing its surface on a polishing pad using an alumina slurry for about $30 \mathrm{~s}$ and sonicated in an ultrasonic bath for about $5 \mathrm{~min}$. A thin coating of the porphyrin catalyst was introduced onto the cleaned electrode surface by dipping it into the coating solution. Next, the solvent of the coating was allowed to evaporate, and the coated electrode was placed in the electrolyte solution for several min before use. Subsequent coatings were made after cleaning the coated electrode with a few drops of dichloromethane. 


\section{RESULTS}

Cyclic voltammetric experiments of $5.0 \times 10^{-3} \mathrm{M}$ chloroacetic acid in aqueous $0.1 \mathrm{M}$ $\mathrm{LiCl}$ solution showed no peaks within the working potential range of the bare glassy, carbon electrode. A glassy carbon electrode coated with a thin layer of $\mathrm{Fe}$ (III)TPPCl exhibited a remarkably small background current compared to that of an uncoated electrode. Although the redox behaviour of the $\mathrm{Fe}$ (III)/Fe(II) couple was not apparent at the coated electrode, it showed a reduction peak at $-0.23 \mathrm{~V}$ in the presence of chloroacetic acid (Figure 1). The peak location $(-0.23 \mathrm{~V})$ was found to be independent of the initial potential and of the scan rate. Cyclic voltammetric experiments of the coated electrode conducted at a constant concentration of chloroacetic acid with different scan rates yielded a straight line when $\log \mathrm{i}_{\mathrm{p}}$ was plotted against $\log v$, where $\mathrm{i}_{\mathrm{p}}$ is the peak current and $v$ is the scan rate (Figure 2). Furthermore, cyclic voltammetric experiments were repeated several times with the same coating in order to check its stability (Figure 3).

All amperometric experiments were conducted at a constant potential of $-0.25 \mathrm{~V}$. Sequential additions of the $5.0 \times 10^{-3} \mathrm{M}$ stock solution of chloroacetic acid into the aqueous electrolyte system $(0.125 \mathrm{~mL}$ each time $)$ were made and the amperometric current was monitored. It was observed that the current did not plateau even after 10 injections. Similar amperograms were obtained at different solvent compositions of water and acetonitrile. A sample amperogram and the calibration curves obtained at different compositions are shown in Figures 4 and 5 , respectively.

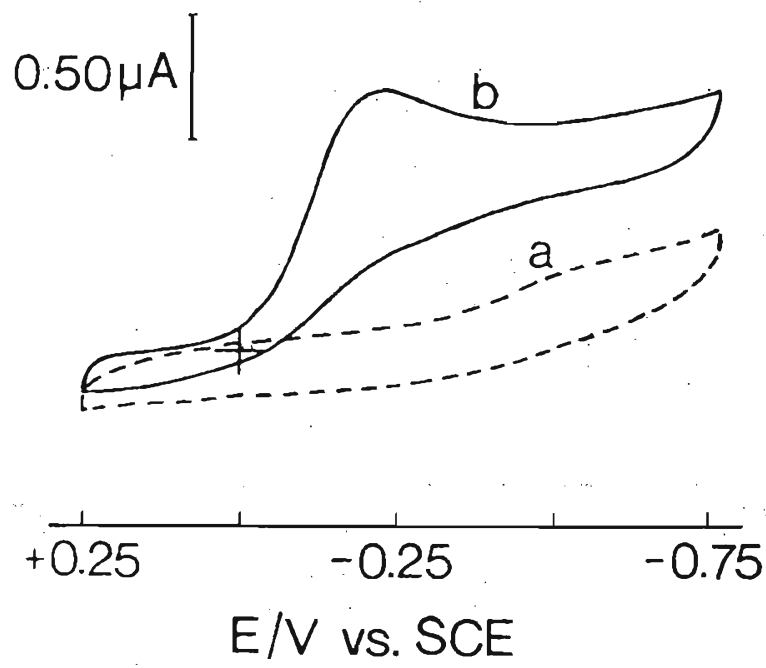

Figure 1: Cyelic volta mmograms of Fe(III)TPPCl coated GC electrode in $\mathrm{H}_{2} \mathrm{O}$ under $\mathrm{N}_{2}$ saturation. Supporting electrolyte $0.1 \mathrm{M} \mathrm{LiCl} \mathrm{in} \mathrm{H}_{2} \mathrm{O}$, scan rate $10 \mathrm{mV} \mathrm{s}$ s. $^{-1}$. (a) with no substrate (b) with $5.0 \times 10^{-3} \mathrm{M}$ chloroacetic acid. 


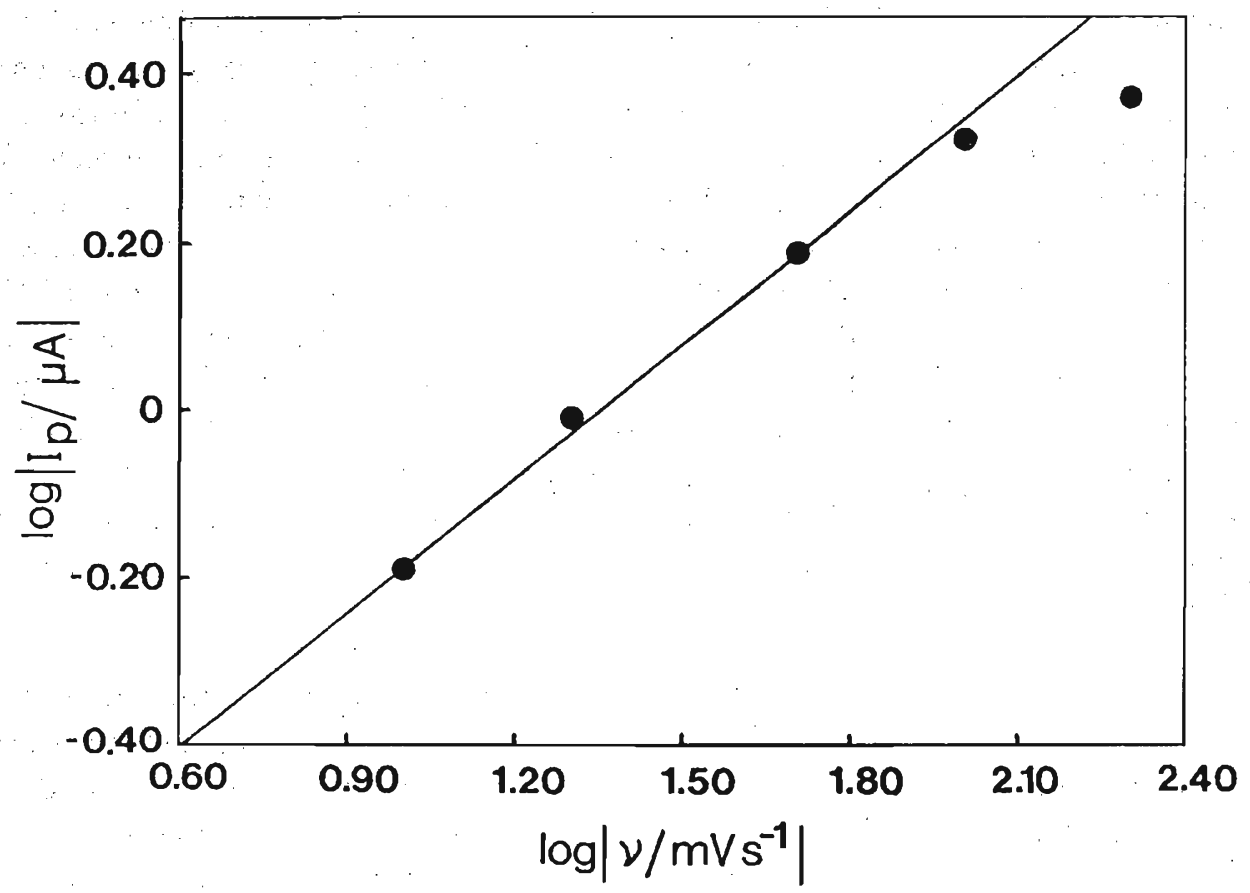

Figure 2: The graph of $\log i_{p}$ vs. $\log v$ for Fe(III)TPPCl coated GC electrode in $5 \times 10^{-3} M$ chloroacetic acid solution under $\mathrm{N}_{2}$ saturation. Supporting electrolyte $0.1 \mathrm{M}$ $\mathrm{LiCl}$ in $\mathrm{H}_{2} \mathrm{O}$.

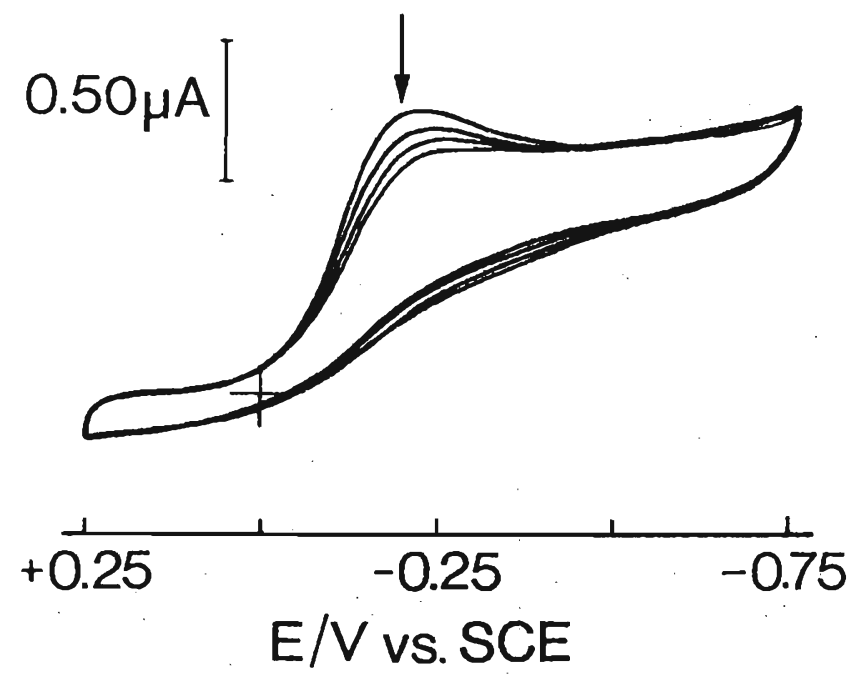

Figure 3: Repeated cyclic voltammograms of Fe(III)TPPCl coated GC electrode in $5.0 \times 10^{-8} \mathrm{M}$ chloroacetic acid dissolved in $\mathrm{H}_{2} \mathrm{O}$ under $\mathrm{N}_{2}$ saturation. Supporting electrolyte $0.1 \mathrm{M} \mathrm{LiCl}$ in $\mathrm{H}_{2} \mathrm{O}$, scan rate $10 \mathrm{mV} \mathrm{s}^{-1}$. The direction of the arrow indicates the subsequent voltammograms. 


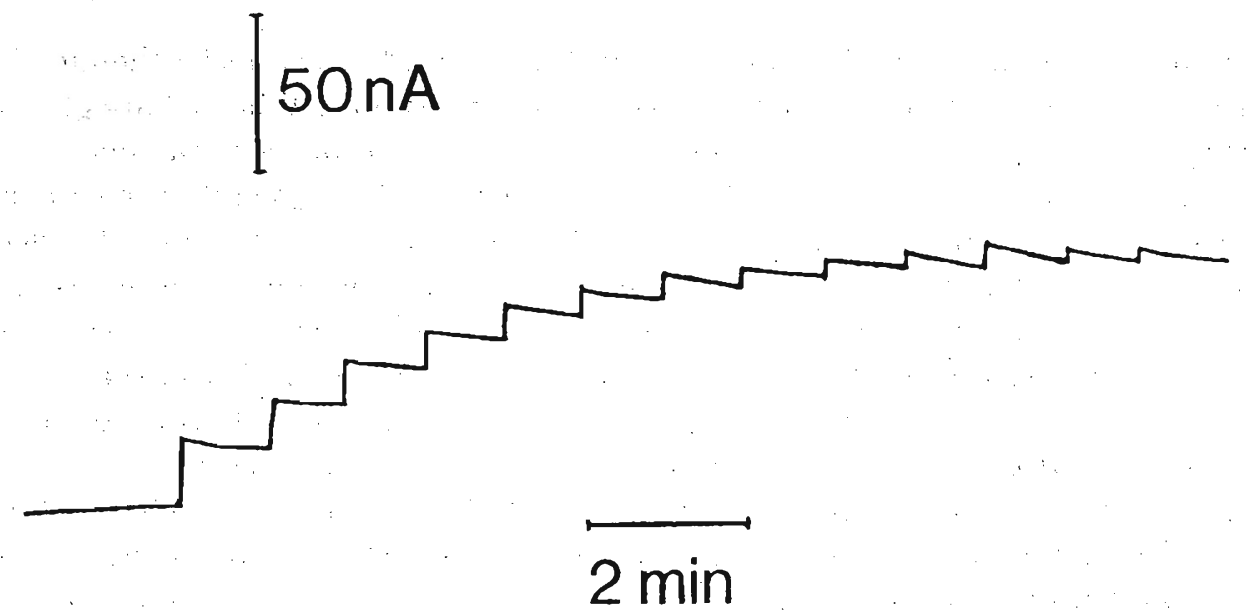

Figure 4: Amperometric current-time responses obtained with Fe(III)TPPCl coated GC electrode with increasing concentration of chloroacetic acid in $25 \mu \mathrm{M}$ steps, under $\mathrm{N}_{2}$ saturation. Applied potential $\mathbf{0 . 2 5} \mathrm{V}$, supporting electrolyte $0.1 \mathrm{M} \mathrm{LiCl}$ in $\mathrm{CH}_{3} \mathrm{CN} / \mathrm{H}_{2} \mathrm{O}(1: 7)$.

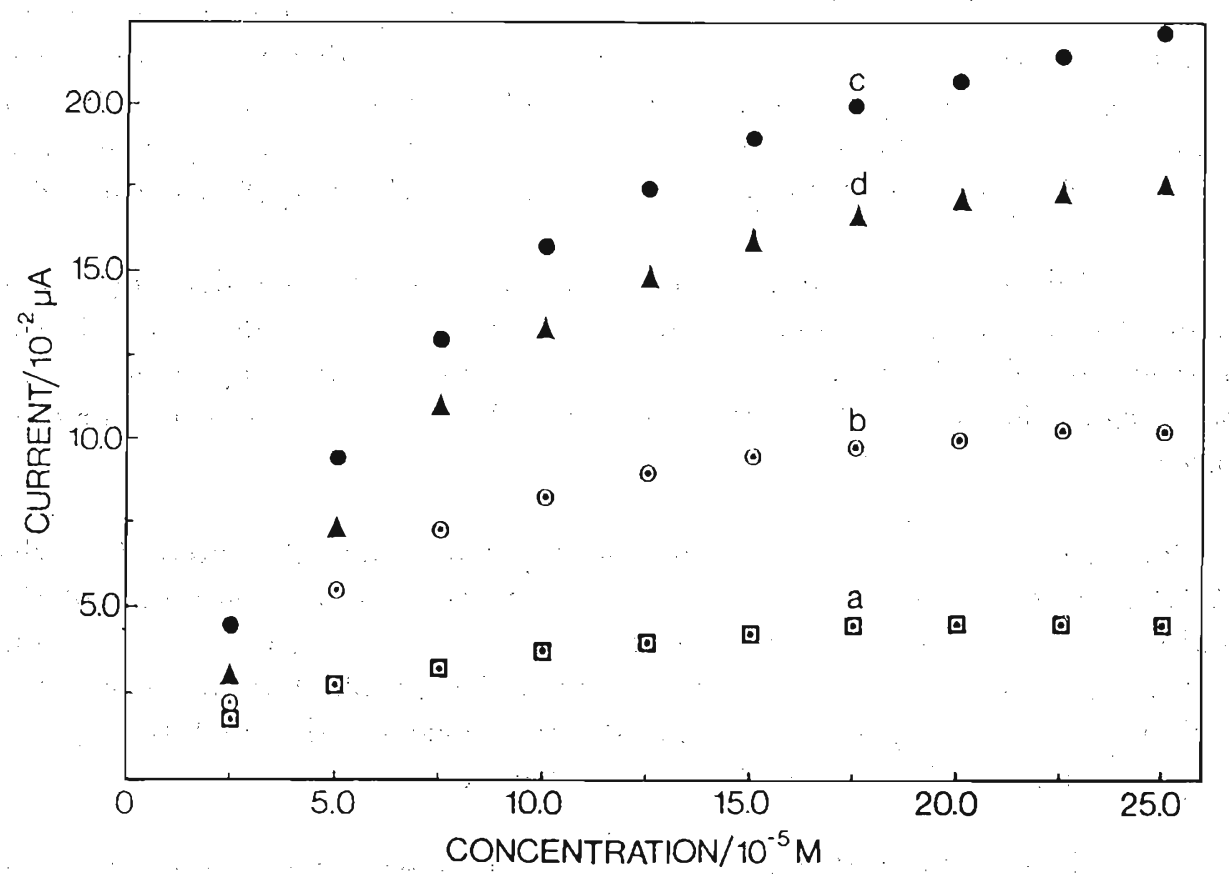

Figure 5: Calibration curves for Fe(III)TPPCl coated GC electrode under $\mathrm{N}_{2}$ saturation in $0.1 \mathrm{M} \mathrm{LiCl}$ at different solvent compositions of $\mathrm{CH}_{3} \mathrm{CN} / \mathrm{H}_{2} \mathrm{O}$ (a) 0:1 (b) 1:7 (c) $1: 3$ (d) $3: 5$. 


\section{DISCUSSION}

Voltammetric experiments show that chloroacetic acid is not electrochemically active in aqueous medium at bare glassy carbon electrodes. When a bare glassy carbon surface is coated with the Fe(III)TPPCl solution, several layers of Fe(III)TPPCl molecules are deposited on the surface. We suggest that the innermost layer is chemically adsorbed while the other layers are weakly bonded (physical adsorption). Bare electrodes on which a catalytic material is deposited display catalytic properties of the incorporated substance. For instance, Fe(III)TPPCl coated electrodes can be used as catalytic electrodes for reduction of organohalides. Such electrodes were used for the heterogeneous electrocatalytic reduction of chloroacetic acid in aqueous medium. Similar heterogeneous processes have also been reported by several researchers. ${ }^{9,14}$ It would be impossible to carry out this catalytic reduction as a homogeneous process, because the Fe(III)TPPCl catalyst is insoluble in aqueous medium.

We suggest that the catalytic reduction process of chloroacetic acid observed at $-0.23 \mathrm{~V}$ (Figure 1) follows the ECE mechanism; where the reduction of $\mathrm{Fe}$ (III)TPPCl to Fe(II)TPP is the first electrochemical step. The catalytically active $\mathrm{Fe}$ (II) form of the porphyrin then reacts chemically with chloroacetic acid resulting in the Fe(III)TPP.chloroacetic acid adduct. Formation of similar adducts and possible reaction mechanisms have already been reported between the reduced form of a metalloporphyrin and organo halides. ${ }^{14,16-18}$ During the third step, the adduct undergoes another electroreduction step leading to various reduction products of chloroacetic acid. However, no attempt was made to identify the products of the reaction as it was not the goal of this research.

The catalytic peak current - scan rate relationship was studied in order to understand the modes of mass transfer of substrate molecules towards the electrode surface. The slope of 0.5 for the $\log i_{p} v s . \log v$ graph suggests that the above process is controlled by diffusion because $i_{p}$ is proportional to $v^{1 / 2}$ for such processes. ${ }^{19}$

Repeating cyclic voltammograms does not decrease the current of the catalytic peak by a significant extent showing the chemical stability of the modified electrode. Electrodes prepared by evaporating a droplet of a coating solution on the surface (droplet evaporation) are less stable than those prepared by dipping in a coating solution (dip-coating). Consequently, the latter type is recommended for all amperometric measurements.

The constant potential of $-0.25 \mathrm{~V}$ vs. SCE was selected for all amperometric studies as it is close to the potential of the highest catalytic current. Although higher amperometric currents may be obtained at more negative applied potentials, some interfering species, including oxygen, would undergo reduction 
reactions. On the other hand, amperometric currents at less negative potentials would result in lower sensitivities. Thus, the potential of $-0.25 \mathrm{~V}$ is a compromise between the catalytic current and interferent problems. Therefore, it is not necessary to conduct further amperometric experiments in order to optimize the applied potential.

Other parameters such as the electrolyte and the coating thickness were not optimized as they have been predetermined..$^{20}$ However, the solvent composition of the medium has to be optimized because it may depend on the chemical nature of the analyte.

The catalytic current at each addition of chloroacetic acid was estimated by extrapolating the background current, and a calibration plot of amperometric current vs. bulk concentration of chloroacetic acid was constructed at each solvent composition. In order to determine the concentration of chloroacetic acid of a given sample, an appropriate amount of the sample is added to the electrochemical cell and the resulting amperometric current is measured. The concentration of chloroacetic acid associated with the measured amperometric current can be obtained from the calibration plot. Although the sensitivity of detection is a little higher in mixed solvent systems, lower and upper detection limits in pure water and in mixed solvent systems are in the same order of magnitude. Further, reproducible results are obtained in all solvent systems.

\section{Acknowledgement}

Financial support from NARESA (RG/94/C/01) is acknowledged.

\section{References}

1. Das K.G. (1981). Pesticide analysis p. xiii. Marcel Dekker, New York.

2. McGonigle E.J. \& Bigley F. (1985). Modern practice of gas chromatography (Ed. R.L. Grobe) pp 775. Wiley, New York.

3. Borman S.A. (1982). New electroanalytical pulse techniques. Analytical Chemistry 54(6): 698A-705A.

4. Johnson D.C. \& Polta T.Z. (1986). Amperometric detection in liquid chromatography with pulsed cleaning and reaction of noble metal electrodes. Chromatography Forum 37-43.

5. Bettelheim A., White B.A. \& Murray R.W. (1987). Electrocatalysis of dioxygen reduction in aqueous acid and base by multimolecular layer films of electropolymerized cobalt tetra(o-aminophenyl)porphyrin. Journal of Electroanalytical Chemistry 217: 271-286. 
6. Widelov A. (1993). Pyrolysis of iron and cobalt porphyrins sublimated onto the surface of carbon black as a method to prepare catalysts, for $\mathrm{O}_{2}$ reduction. Electrochimica Acta 38(17): 2493-2502.

7. Lexa D., Saveant J.M. \& Wang D.L. (1986). Electroreductive alkylation of iron porphyrins. Iron(III), iron(II), and iron(I) alkyl complexes from the reaction of doubly reduced iron(II) porphyrins with alkyl halides. Organometallics 5(7): 1428-1434.

8. Che G. \& Dong S. (1993). Application of an ultramicroelectrode in homogeneous catalytic reactions - PartIV. Electrocatalytic reduction of organohalides by metalloporphyrin at ultramicroelectrode. Electrochimica Acta 38(10): $1345-1349$.

9. Wang J: \& Golden T. (1989). Metalloporphyrin chemically modified glassy carbon electrodes as catalytic voltammetric sensors. Analytica Chimica Acta 217: 343-351.

10. Pang D.W. \& Wang Z.L. (1993). Electrocatalysis of several water-soluble porphyrins for the oxidation of some small molecules. Journal of Electroanalytical Chemistry 358(7): 235-246.

11. Truxillo L.A. \& Davis D.G. (1975). Electrochemistry of cobalt tetraphenylporphyrin in aprotic media. Analytical Chemistry 47(13): 2260-2267.

12. Galen D.A.V.\& Majda M. (1988). Irreversible self-assembly of monomolecular layers of a cobalt(II) hexadecyltetrapyridylporphyrin amphiphile at gold electrodes and its catalysis of oxygen reduction. Analytical Chemistry 60(15): 1549-1553.

13. Park S.B., Matuszewski W., Meyerhoff M.E., Liu Y.H. \& Kadish K.M. (1991). Potentiometric anion selectivities of polymer membranes doped with indium(III)-porphyrins. Electroanalysis 3: 909-916.

14: Root D.P., Pitz G. \& Priyantha N. (1991). Electrocatalytic metalloporphyrin electrode for detection of organohalides. Electrochimica Acta 36(5/6): 855858.

15. Worthing C.R. \& Walker S.B. The pesticide manual (8th ed). British Crop Protection Council, London. 
16. Lexa D., Saveant J.M. \& Soufflet J.P. (1979). Chemical catalysis of the electrochemical reduction of alkyl halides. Comparison between cobalttetraphenyl porphin and vitamin B12 derivatives. Journal of Electroanalytical Chemistry 100: 159-172.

17. Lexa D., Mispelter J. \& Saveant J.M. (1981). Electroreductive alkylation of iron in porphyrin complexes. Electrochemical and spectral characteristics of $\sigma$-alkyliron porphyrins. Journal of American Chemical Society 103: 6806-6812.

18. Kadish K.M., Lin X.Q. \& Han B.C. (1987). Chloride-binding reactions and electrochemistry of (TPP)Co(II) and (TPP)Co(III)Cl in dichloromethane. Inorganic Chemistry 26(25): 4161-4167.

19. Bard A.J. \& Faulkner L.R. (1980). Electrochemical methods. p. 218. Wiley, New York.

20. Priyantha N. \& Weerabahu D. (1996). Amperometric sensor for propanil. Analytica Chimica Acta 320: 263-268. 